investigators, it is obvious that we need to adopt standard flux values, sizes, and brightness distributions for calibration sources in order to put all measurements on a relative scale. These data are often omitted in final publication of results dealing with the galactic centre region and this slows the reliable comparison of results between groups.

Bolton: The apparent lack of absorption of the southern part of Drake's ring by the 3-kpc arm need not mean that there is necessarily no absorption. The emission background often has considerable fine structure and if such coincides with the direction of the source its effect may be included in the apparent absorption deduced from the subtraction of the composite and "expected" profile. This effect shows quite clearly in absorption profiles deduced from observations with telescopes of different resolving power. Fine structure in the emission background can appear as false absorption, or add to or subtract from true absorption effects.

\title{
46. INTERPRETATION OF VELOCITY DISTRIBUTION OF THE INNER REGIONS OF THE GALAXY
}

\author{
G. DE VAUCOUleurs \\ University of Texas
}

I. The large positive and negative velocities in the $21-\mathrm{cm}$ line profiles near the galactic centre have indicated the presence of substantial departures from circular motions in the central parts of the Galaxy. The Leiden astronomers (Oort and Rougoor 1958; Rougoor and Oort 1960) have interpreted these observations in terms of an "expanding arm" at a mean distance of about $3 \mathrm{kpc}$ from the centre. It is not clear how these arms or arcs are related to the regular spiral structure, if the Galaxy is an ordinary spiral similar to M31 as commonly assumed. If, on the other hand, the Galaxy is similar to the $\mathrm{SAB}(\mathrm{r})$ or $\mathrm{SAB}(\mathrm{rs})$ systems, as suggested by the multiplicity of the spiral pattern discussed in another communication, a different interpretation of the velocity distribution is possible.

II. The average diameter of the rings in $\mathrm{SB}(\mathrm{r})$ spirals of the Virgo cluster is $1: 6=5 \cdot 0$ to $6 \cdot 3 \mathrm{kpc}$, if the distance is 10 to $12 \cdot 6 \mathrm{Mpc}\left(m_{0}-M=30 \cdot 0\right.$ to $30 \cdot 5$; de Vaucouleurs 1957); it ranges from 2 to $4 \mathrm{kpc}$ for the other ringed types. This value agrees well with the 3 -kpc radius of the "expanding arm" derived from the tangential point, and it confirms the presence of a ring structure in the central region. Since the ring seems to be incomplete an open ring structure as in the $\mathrm{SB}(\mathrm{rs})$ and $\mathrm{SAB}(\mathrm{rs})$ types is suggested; the length of the bar is tilted about 30 to $45^{\circ}$ to the Sun-centre line with the breaks of the ring roughly at right angles to the bar (cf. Fig. 1).

In this model the negative velocities in the direction of the centre are interpreted not as an expanding arm or ring, but as indicating that the gas is streaming along the bar from the nucleus outward at velocities of the order of 60 to $70 \mathrm{~km} / \mathrm{sec}$, the line-of-sight component being $53 \mathrm{~km} / \mathrm{sec}$ in the direction of the nucleus (Oort, Kerr, and Westerhout 1958). Such a flow pattern, which is strongly suggested by the narrow dust lanes commonly observed along the bars of barred spirals, was directly detected by recent spectroscopic observations of several systems described in another communication.

III. A frequent characteristic of barred spirals is the small, elliptical, and often exceedingly bright and sharp nucleus, e.g. as in NGC 1365, 1433, 4303, and 5236. 
Such nuclei appear almost as singular points detached from the rest of the structure. In the present model, with gas streaming along the bar symmetrically away from the centre, it is tempting to regard such nuclei as the foci of convergence, or injection points of the in-falling gas from the galactic corona. Each volume element of the gas must be rotating with the velocity appropriate to its $r$ and $z$ components. The motion may be visualized as a double vortex funnelling coronal matter toward the nucleus. This vortical motion might be looked for by a detailed study of $21-\mathrm{cm}$ line profiles at intermediate latitudes within 10 to $20^{\circ}$ from the centre in longitude.

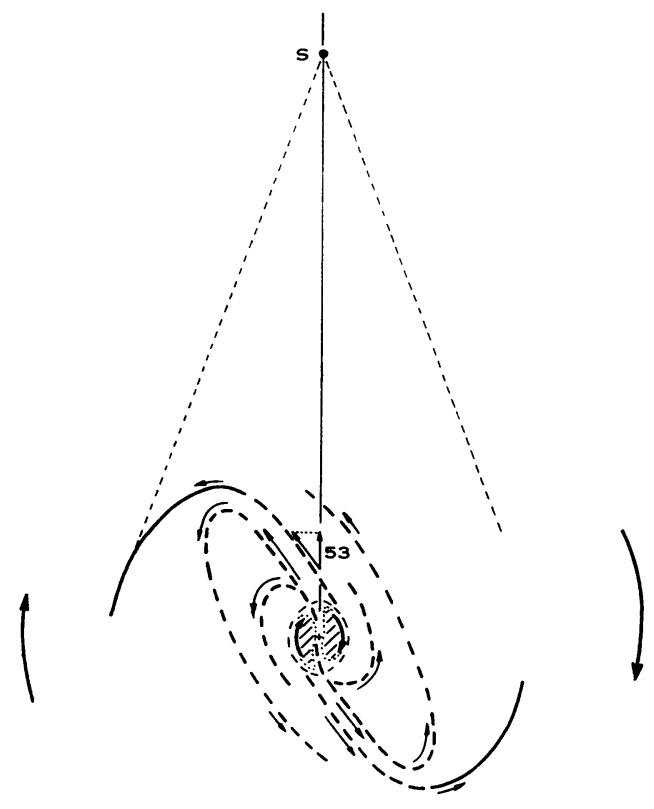

Fig. 1.-Possible structure of inner regions of the Galaxy consistent with SAB(rs) structure of outer regions. Compare with photograph of NGC 4303. Dashes show main gas and dust streams superimposed on general rotation.

The in-falling of the gas need not be limited to the nuclear region but may occur with progressively reduced intensity (both as to density and velocity) at increasing distances from the centre. Possibly the low-intensity components of the $21-\mathrm{cm}$ lines observed in high galactic latitudes at predominantly negative velocities (McGee and Murray 1961) are, at least in part, evidence for the postulated circulation (cf. Fig. 2).

IV. The bulk of the gas in the low-density corona should be ionized, and the vortical motion may be strengthened if, in addition to the gravitational field, a magnetic dipole field with its N.-S. axis along the axis of rotation of the Galaxy is present in the region of the nucleus. The focalized vortices plunging toward the nucleus from both sides of the galactic plane are somewhat similar to two rotating plasmoids that are shot at each other for a head-on collision (Bostick 1958). Depending on the strength of the magnetic field and the injection velocity, actual collision may 
or may not take place; it is known that relative line intensities in emission spectra of galactic nuclei (Seyfert 1943; E. M. Burbidge and G. R. Burbidge 1962) are sometimes indicative of collisional, rather than radiative, excitation. In general it may be presumed that magnetic forces will effectively prevent actual collision or limit its extent, and that most of the gas will be sharply deflected in two streams (recombining to neutral upon reaching the higher density in the galactic plane) flowing in opposite directions away from the nucleus. If so, a permanent flow regime will be set up along the bar under the constant pressure of the in-falling coronal gas.

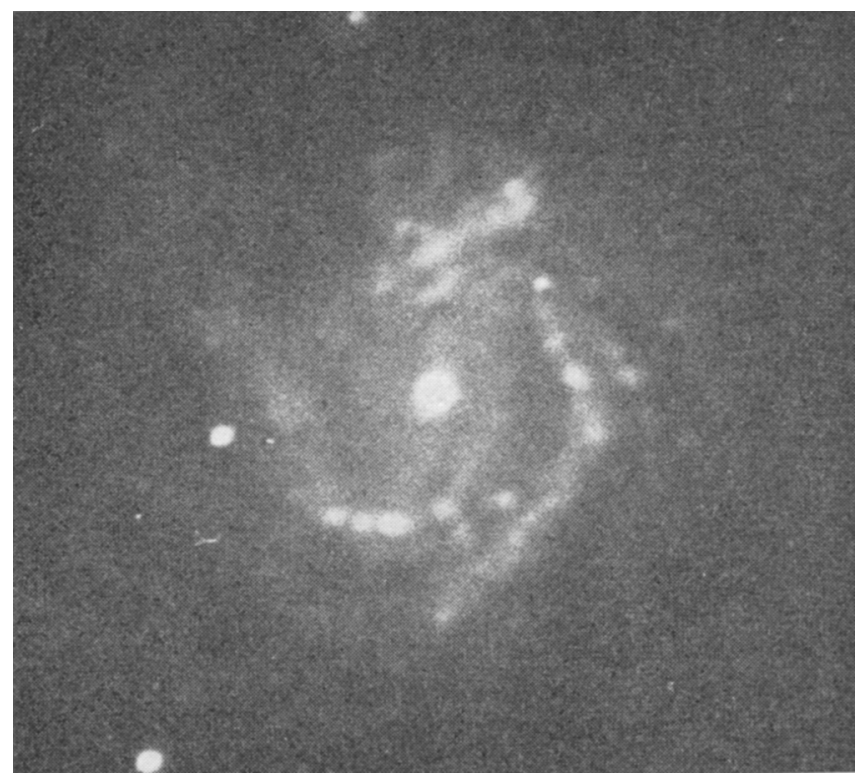

Fig. 2.-Inner regions of NGC 4303 (82-inch reflector, McDonald Observatory).

V. Because most of the interstellar gas injected into the system will be eventually removed by star formation, only a small fraction at best might be involved in a closed-circuit or recirculating flow pattern; rather one might postulate that the influx of gas from the corona required to maintain a steady centrifugal flow along the bar and into the arms is supplied and driven by the condensation of primordial intergalactic gas, perhaps as in the hypothesis of Gold and Hoyle (1959) on galaxy formation.

At any rate it is probable that the gas vortices reach their maximum velocity at the injection points, i.e. in the nucleus. This is supported by the surprisingly high rotational velocities observed optically in galactic nuclei (Lallemand, Duchesne, and Walker 1960; E. M. Burbidge, G. R. Burbidge, and Prendergast 1962) and at $21 \mathrm{~cm}$ in the nucleus of the Galaxy (Rougoor and Oort 1960). The current interpretation of the latter as a "rotating ring" postulates a structure not commonly present in other galaxies. 
An atom injected in the bar with an initial velocity $V_{0}$ and moving away from the centre is slowed down by the central attractive force of the mass $M(r)$ within its distance $r$ to the centre; the stream velocity $V_{s}$ is then expected to decrease, at first slowly, then more and more rapidly, and to become negligible, i.e. of the order of the turbulent velocities; say, $\approx 20 \mathrm{~km} / \mathrm{sec}$, at some distance $r_{s}$, probably equal to the radius of the ring structure, where gravitation and differential rotation of the stellar system as a whole become the dominant forces acting on the gas. There is some direct evidence, for instance in the Large Magellanic Cloud (de Vaucouleurs 1960; Feast, Thackeray, and Wesselink 1961) that the angular velocity of rotation is constant in the region of the bar; in fact a bar structure could probably not develop and persist against the shearing forces of a large differential rotation.

VI. This model may help also to account for the presence of an extended radio source in and near the galactic nucleus. The length of the source is 4 to 5 degrees in longitude (Mills 1956,1959 ) or approximately 0.5 to $0.7 \mathrm{kpc}$. For comparison the sharp, elliptical nucleus of the SB(r) types is about $0.5 \mathrm{by} 1.0 \mathrm{kpc}$; for the assumed tilt of the bar to the line of sight in the Galaxy the projected diameter would be 0.5 to $0.6 \mathrm{kpc}$. In view of the assumed injection mechanism involving glancing collision of plasma vortices the presence of enhanced continuous radio emission in the nucleus is not surprising.

\section{References}

Bostick, W. H. (1958).—Rev. Mod. Phys. 30 : 1090-4=Symp. IAU 8: 1090-4. [Cambridge, Mass. 1957.]

Burbidge, E. M., and Burbidge, G. R. (1962).-Ap. J. 135 : 694-710.

Burbidge, E. M., Burbidge, G. R., and Prendergast, K. H. (1962).-Ap. J. 136: $128-32$.

Feast, M. W., Thackeray, A. D., and Wesselink, A. J. (1961).-M.N. 122: 433-53.

Gold, T., and Hoyle, F. (1959)._-“Paris Symposium on Radio Astronomy." (Ed. R. N. Bracewell.) Symp. IAU 9: 583-8. [Paris 1958.] (Stanford Univ. Press.)

Lallemand, A., Duchesne, M., and Walker, M. F. (1960).-P.A.S.P. 72: 76-84.

McGee, R. X., and Murray, J. D. (1961).-Aust. J. Phys. 14: 260-78.

Mills, B. Y. (1956).-Observatory 76: 65-7.

Mills, B. Y. (1959).-P.A.S.P. 71 : 267-91.

Oort, J. H., KerR, F. J., and Westerhout, G. (1958).-M.N. 118 : 379-89.

Oort, J. H., and Rougoor, G. W. (1958): Kon. Nederl. Akad. Wet. Amsterdam $67: 139-43$.

Rougoor, G. W., and Oort, J. H. (1960).-Proc. Nat. Acad. Sci. (Washington) 46: 1-13.

SEYFERT, C. K. (1943).-Ap. J. $97: 28-40$.

DE VAUCOUleurs, G. (1957).-A.J.62: 13.

DE VAUCOUleurs, G. (1960).-Ap. J. $131: 265-81$.

\section{Discussion}

Oort: Have you any direct observational evidence for expanding motions along bars?

de Vaucouleurs: Yes, we have spectrographic observations showing these motions (see this volume, paper 58).

Oort: You can distinguish between rotation and expansion motions?

de Vaucouleurs: Yes, and most easily when the bar is along the minor axis. Also the rotation velocities of the bars are relatively low.

Aller: In order to study such phenomena as possible flow of gas along the bars in barred spirals, it is necessary to have conditions of exquisite seeing and adequate scale. The technique so successfully developed by M. Walker at the Lick Observatory involves using an image con- 
verter at the coude spectrograph of the 120 -inch reflector. It would be of great value if this or similar equipment could be applied to central regions of barred spirals. I am not aware that Walker has made any measurements of barred spirals.

Ambartsumian: How large are the velocities of streaming you have observed in barred spirals? Have you observed the discontinuity of radial velocity near the central part of the bar?

de Vaucouleurs: The actual values $(\sim 50 \mathrm{~km} / \mathrm{sec})$ are difficult to determine because of scale problems - the scale at the prime focus of the 82 -inch is very small for this work. However, the lines are definitely inclined along the bar. This evidence will be presented tomorrow.

Thackeray: Did I understand you to say that you have cases of a bar pointing directly towards us and, if so, how do you know this?

de Vaucouleurs: Yes, there are definite cases such as NGC 55 and 4631, which are indicated by studies of luminosity distribution and spectrographic observations (see Ap.J. 133 : 405-12 (1961)).

Blaauw: Stating that the observed velocities along the bar are outward, not inward, implies that you know the direction of tilt of the system. Is that well established?

de Vaucouleurs: In some, the direction of tilt is clearly indicated; in others, one can argue about the sign. In edge-on systems, the question does not arise.

\title{
47. DISTRIBUTION DES VITESSES RADIALES DES REGIONS HII DANS LA DIRECTION DU CENTRE GALACTIQUE
}

\author{
G. Courtes
}

Observatoire de Marseille

\section{Introduction}

La Station Française de Zeekoegat (Province du Cap-Afrique du Sud), construite dans le cadre de l'Observatoire Européen Austral est consacrée à l'étude des vitesses radiales par le prisme objectif de Fehrenbach. Parallèlement aux observations du prisme objectif, nous avons, avec P. Cruvellier, conduit un programme d'observations des régions HII de l'Hémisphère Sud.

(a) L'intérêt essentiel des recherches de régions HII est, du point de vue des méthodes d'observation:

$1^{\circ}$-leur caractère monochromatique (émission $\mathrm{H} \alpha$ ),

$2^{\circ}$-leur diamètre apparent considérable (surfaces étendues).

Le premier de ces caractères autorise une sélection aussi grande que l'on veut avec une élimination quasi parfaite du continu.

Le deuxième offre la possibilité d'utiliser les plus grands rapports d'ouverture et d'augmenter ainsi dans un facteur considérable l'éclairement de la plaque.

L'exploitation de ces deux caractères 1 et 2 donne la possibilité de découvrir des régions HII extrêmement faibles ou très absorbées. Le caractère monochromatique donne la possibilité de connaître avec une grande précision la vitesse radiale et les mouvements internes des régions HII; grâce à l'utilisation de l'étalon interférentiel de Perot et Fabry.

(b) Du point de vue purement astrophysique, l'intérêt essentiel des régions HII est leur remarquable répartition régulière le long des bras spiraux (M33, M31, M51, etc. . . .) qui en font l'un des meilleurs guides dans les recherches de structure de la Galaxie, comme l'avaient si bien remarqué Baade et Morgan (Courtès 1962b, 1963b). 